\title{
PARÂMETROS DO TESTE DE ENVELHECIMENTO ACELERADO PARA DETERMINAÇÃO DO VIGOR DE SEMENTES DE FEIJÃO ${ }^{1}$
}

\author{
DANILA COMELIS BERTOLIN² ${ }^{2}$ MARCO EUSTÁQUIO DE SÁ3 ${ }^{3}$ ERICA RODRIGUES MOREIRA²
}

RESUMO - O vigor de sementes representa atributos da qualidade fisiológica não revelados pelo teste de germinação. O teste de envelhecimento acelerado é realizado em condições de elevadas temperaturas e umidade relativa do ar por períodos curtos, logo estudos a respeito da utilização de temperaturas de 43 a $45^{\circ} \mathrm{C}$ e a redução do período de envelhecimento são possibilidades que merecem reflexão. No estudo foi objetivo a análise dos efeitos de temperaturas e períodos de exposição ao teste de envelhecimento acelerado para identificar o vigor de sementes de feijão e características relativas ao genótipo. O delineamento experimental foi o inteiramente casualizado, foram utilizados 20 genótipos de feijão, sendo que as sementes foram submetidas a cinco períodos de envelhecimento acelerado (12, 24, 36, 48 e $60 \mathrm{~h})$ e três temperaturas $\left(41,43\right.$ e $\left.45^{\circ} \mathrm{C}\right)$. Foram avaliados também germinação, primeira contagem de germinação, velocidade de germinação e emergência de plântulas. Os genótipos de sementes não diferiram quanto ao teste de germinação, no entanto apresentaram níveis distintos de qualidade através do teste de envelhecimento acelerado. O teste de envelhecimento acelerado é conveniente quando realizado a $41^{\circ} \mathrm{C} / 48 \mathrm{~h} \mathrm{e} 43^{\circ} \mathrm{C} / 24 \mathrm{~h}$. É possível a realização do teste de envelhecimento acelerado com utilização de temperatura superior e redução do período de exposição ao teste.

Termos para indexação: Phaseolus vulgaris L., temperatura, germinação, vigor.

\section{PARAMETERS OF THE ACCELERATED AGING TEST TO DETERMINE THE VIGOR OF DRY BEAN SEEDS}

\begin{abstract}
The germination test does not demonstrate the physiological quality attributes of seeds. The accelerated aging test is conducted in conditions of high temperatures and relative humidities for short time periods and, therefore, studies on using temperatures of 43 to $45{ }^{\circ} \mathrm{C}$ and reducing the period of aging deserve to be studied. The objective in this study was to analyze the effects of temperatures and periods of exposure during the accelerated aging test to identify bean seed vigor. The experimental design was of completely randomized blocks with the seeds subjected to five periods of accelerated aging $(12,24,36,48$ and $60 \mathrm{~h})$ and three temperatures (41, 43 and $45{ }^{\circ} \mathrm{C}$ ). Germination, first count, speed germination index and seedling emergence were evaluated. Seed samples did not differ in the germination test, but showed different quality levels from the accelerated aging test, which should be performed at $41{ }^{\circ} \mathrm{C} / 48 \mathrm{~h}$ and $43{ }^{\circ} \mathrm{C} / 24 \mathrm{~h}$. The accelerated aging test can be performed at a higher temperature and for a shorter test period.
\end{abstract}

Index terms: Phaseolus vulgaris L., temperature, germination seed, vigor.

${ }^{1}$ Submetido em 26/08/2009. Aceito para publicação em 26/10/2010. Parte da Tese de Doutorado em Sistemas de Produção, pela primeira autora.

${ }^{2}$ Engenheira Agrônoma, MSc., Bolsista FAPESP, Doutoranda em Sistemas de Produção pela UNESP, campus de Ilha Solteira, Cx. Postal 31, Ilha
Solteira, SP, CEP. 15385-000. E-mail:danilacomelis@hotmail.com .

${ }^{3}$ Engenheiro Agrônomo, Ph. D., Professor da Unesp, campus de Ilha Solteira, Cx. Postal 31, Ilha Solteira, SP, CEP. 15385-000 


\section{INTRODUÇÃO}

O Brasil é o maior produtor de feijão do mundo com produção de $3.588 .200 \mathrm{t}$ e produtividade de $946 \mathrm{~kg}$ $\mathrm{ha}^{-1}$, produtividade considerada baixa de acordo com o potencial produtivo da cultura, sendo que no Distrito Federal, região que apresenta maior produtividade Nacional a produtividade média é de $2,2 \mathrm{t} \mathrm{ha}^{-1}$ (CONAB, 2009). Dentre os fatores à que se atribui a baixa produtividade no país estão o cultivo intensivo em área com a mesma cultura, o preparo inadequado do solo, a falta de adubação equilibrada, a ocorrência de doenças, baixo investimento em tecnologia entre outros, sendo apontado como principal fator a baixa taxa de utilização de sementes melhoradas (Rengel 2005, Yokoyama et al. 2000).

A qualidade das sementes influencia fortemente o sucesso ou fracasso da cultura, especialmente em condições de estresse ambiental, para tanto métodos experimentais de determinação de vigor e germinação, foram desenvolvidos para minimizar o risco de utilização de sementes de baixa qualidade (Halmer, 2000).

O teste padrão germinação é conduzido sob condições favoráveis, que basicamente permite que o lote de sementes expresse sua capacidade máxima. Quando as condições de campo são ótimas, o teste padrão de germinação pode predizer corretamente o desempenho do lote em campo. Na maior parte dos casos, porém, o resultado do teste de germinação superestima os valores reais da emergência de plantas em campo. Estas deficiências levaram a observação de que nem todas as facetas da qualidade das sementes foram devidamente identificadas pelo teste de germinação. Qualquer um dos eventos que antecedem a perda de germinação poderia servir como uma base para testes vigor, por isso um teste de vigor é um índice de qualidade de sementes mais sensível do que o teste padrão de germinação (Copeland e McDonald, 2001).

Um teste de vigor é determinado sob condições desfavoráveis, ou medindo-se o declínio de alguma função bioquímica ou fisiológica (Sá, 1994) e de acordo com McDonald (1980) deve ser: econômico, rápido, simples, objetivo, reprodutível, apresentar resultados que se correlacionem com o desempenho em campo.

$\mathrm{O}$ teste de envelhecimento acelerado integra muitas das importantes características desejadas em um teste de vigor, é rápido, econômico, simples e útil para todas as espécies (Copeland e McDonald, 2001). Inicialmente proposto como um método para avaliar o potencial de armazenamento de sementes, este teste é realizado em condições de alta temperatura e umidade relativa do ar (cerca de 100\%) por períodos curtos (3 a 4 dias), seguido por um teste de germinação (Copeland e McDonald, 2001; Halmer, 2000). As diferenças de umidade inicial das sementes devem ser consideradas na interpretação deste teste (Copeland e McDonald, 2001), logo sementes mais úmidas sofrerão maiores efeitos deletérios do estresse, devido à intensificação da sua atividade metabólica.

$\mathrm{Na}$ prática, o teste é bem sucedido para algumas espécies, como milho, feijão, soja, sorgo entre outras (Marcos Filho, 1999), apesar da evidência de que as sementes apresentem alteração após serem submetidas às condições do teste, em termos de parâmetros celulares básicos, tais como ácidos nucleicos e membranas (Halmer, 2000). De acordo com Dias e Marcos Filho (1995), a exposição das sementes à temperatura e umidade elevadas provoca sérias alterações degenerativas no metabolismo da semente, desencadeando a desestruturação e perda da integridade do sistema de membranas celulares. De acordo com Santos et al. 2004, a partir de 72 horas de envelhecimento artificial para sementes de feijão há redução na atividade das enzimas fosfatase ácida e esterase. Os mesmos autores salientam que o período de envelhecimento e a qualidade inicial dos lotes influencia a atividade das enzimas fosfatase ácida, malato desidrogenase, glumato desidrogenase e esterase, sendo que os lotes com alta germinação podem apresentar acentuadas diferenças em resposta ao processo de envelhecimento como redução na capacidade seletiva das membranas das sementes e pelo crescimento e desenvolvimento das plantas de feijoeiro. Também de acordo com Binotti et al. 2008, no teste de envelhecimento acelerado para sementes de feijão há uma queda expressiva na germinação e vigor, além do elevado aumento no conteúdo de lixiviados, sendo estes constituídos em grande parte de aminoácidos, açúcares, íons de potássio e fósforo, de maneira que o aumento das quantidades de lixiviados está relacionado com o declínio na germinação e vigor de sementes.

Dutra e Teófilo (2007), estudando temperaturas de 40,42 e $45^{\circ} \mathrm{C}$, por $24,48,72$ e $96 \mathrm{~h}$ em dois genótipos de feijão caupi concluíram que a combinação de $42{ }^{\circ} \mathrm{C}$ por $48 \mathrm{~h}$ foi a mais adequada para a avaliação do potencial fisiológico das sementes.

Paiva et al. (2008) realizando envelhecimento acelerado em sementes de forrageira a 41 e $45{ }^{\circ} \mathrm{C}$ com períodos de exposição ao teste por 48,72 e 96 h, 
observaram que quando da utilização da temperatura de 45 ${ }^{\circ} \mathrm{C}$ ocorreram as menores porcentagens de germinação e que o teste pode ser realizado com resultados satisfatórios tanto à $41^{\circ} \mathrm{C}$ por $72 \mathrm{~h}$, quanto à $45^{\circ} \mathrm{C}$ por $48 \mathrm{~h}$.

Alguns estudos são encontrados na literatura para teste de envelhecimento acelerado em sementes de feijão sendo a maioria com temperaturas abaixo de $42^{\circ} \mathrm{C}$ (Scappa Neto et al. 2001, Binotti et al. 2008, Mello e Tillmann, 1987), assim a utilização de temperaturas de 43 a $45{ }^{\circ} \mathrm{C}$ e a redução do período de envelhecimento são possibilidades que merecem reflexão (Marcos Filho, 1999), tendo em vista as características desejáveis em um teste de vigor. Com base nas necessidades de maiores estudos a respeito deste teste de vigor, neste estudo objetivou-se verificar os efeitos de temperaturas e períodos de exposição ao teste de envelhecimento acelerado para identificar o vigor de sementes de feijão e a sensibilidade dos genótipos em relação ao estresse imposto pelo teste.

\section{MATERIAL E MÉTODOS}

O experimento foi realizado no Laboratório de Análise de Sementes do Departamento de Fitotecnia, Economia e Sociologia Rural, da Faculdade de Engenharia da UNESP - Campus de Ilha Solteira durante o período de março a junho de 2009. Foram conduzidos testes de germinação, primeira contagem de germinação, velocidade de germinação e de emergência de plantas em campo com a finalidade de se comparar as informações fornecidas pelo teste de envelhecimento acelerado. Utilizaram-se seis cultivares Juriti, Talismã, Colibri, Pérola, Rubi e IAC-Carioca, e uma linhagem, LP 04-92, cultivadas no Estado de São Paulo e provenientes da safra de 2008. Os testes seguiram os seguintes procedimentos: Teste de germinação: foi realizado utilizando-se de quatro repetições de 50 sementes para cada cultivar utilizada. As sementes foram distribuídas em papeltoalha e colocadas para germinar em Germinador do tipo Mangelsdorf regulado para manter a temperatura constante de $25{ }^{\circ} \mathrm{C}$. A condução dos testes seguiu as Regras para Análise de Sementes (Brasil, 2009). Foram computadas as porcentagens de plântulas normais para cada repetição, obtendo-se os valores médios por cultivar, sendo que as contagens foram efetuadas no $5^{\circ}$ e $9^{\circ}$ dia após a instalação do teste; Primeira contagem de germinação: foi realizado em conjunto com o teste de germinação, computando-se as porcentagens de plântulas normais verificadas no $5^{\circ}$ dia; Velocidade de germinação: foi realizada em conjunto com o teste de germinação computando-se as plântulas normais durante o período de avaliação do teste padrão de germinação. Com os dados obtidos, o índice de velocidade para cada repetição foi calculado segundo a expressão proposta por Maguire (1962), onde: $\mathrm{VG}=+\underline{\mathrm{N} 1}+\underline{\mathrm{N} 2}+\underline{\mathrm{Nn}}$ tendo-se: $\overline{\mathrm{D} 1} \overline{\mathrm{D} 2} \mathrm{\textrm {Dn }}$

$\mathrm{VG}=$ velocidade de germinação; N1, N2, ..., Nn = número de plântulas germinadas a 1,2 e $\mathrm{n}$ dias após a semeadura, respectivamente; D1, D2, ..., Dn = número de dias após a implantação do teste; Emergência das plantas em campo: foi realizado na Fazenda de Ensino e Pesquisa da UNESP, campus de Ilha Solteira, foram semeadas quatro repetições de 50 sementes, distribuídas em sulcos de $2 \mathrm{~m}$ de comprimento, com $5 \mathrm{~cm}$ de profundidade. As contagens foram realizadas aos 20 dias após a semeadura e os dados foram transformados em porcentagem; Massa de 1000 sementes: determinado utilizando-se 8 subamostras de 100 sementes para cada cultivar. As sementes foram pesadas em balança de precisão 0,001 g, conforme as Regras para Análise de Sementes (Brasil, 2009); Teor de água das sementes: foi determinado pelo método da estufa a $105^{\circ} \mathrm{C}$, por 24 horas, com 2 repetições por cultivar, antes e após a realização do teste de envelhecimento acelerado; Teste de envelhecimento acelerado: foi realizado pelo método gerbox, de acordo com metodologia descrita por Marcos Filho (1994), com $40 \mathrm{~mL}$ de água destilada por gerbox às temperaturas de 41,43 e $45{ }^{\circ} \mathrm{C}$ por períodos de 12,24 , 36,48 e 60 h. Decorrido o período de exposição ao teste as sementes foram submetidas ao teste de germinação (Brasil, 2009) com quatro repetições de 50 sementes para cada tratamento. Os valores médios obtidos para cada material foram expressos em porcentagem. Por ocasião da realização dos testes a quantidade de sementes necessária foi retirada da embalagem, e o restante permanecia armazenado em câmara seca (condições de $20^{\circ} \mathrm{C}$ e $40 \%$ de umidade relativa).

O delineamento experimental utilizado foi o inteiramente casualizado, com 4 repetições. As análises estatísticas foram realizadas pelo Programa SANEST (Zonta e Machado, 1991), constando de análise de variância para verificar se houve diferença entre os tratamentos, teste de Tukey a $1 \%$ e $5 \%$ de probabilidade para comparação de médias e correlação linear simples entre os resultados obtidos para envelhecimento acelerado, germinação e as demais variáveis de vigor (Banzatto e Kronka, 2006). 


\section{RESULTADOS E DISCUSSÃO}

A Tabela 1 apresenta os valores médios para massa de 1000 sementes, teor de água inicial das sementes, germinação e vigor.

As amostras de sementes testadas neste estudo apresentaram diferenças iniciais de teor de água (Tabela 1), no entanto dentro dos limites aceitáveis de acordo com Marcos Filho, 1999.

De acordo com os resultados para germinação, primeira contagem de germinação e velocidade de germinação os genótipos apresentaram potencial semelhante, não diferindo estatisticamente entre si, no entanto diferenças foram evidenciadas nos resultados de emergência de plantas. É conhecida a influência do genótipo sobre o comportamento fisiológico das sementes (Marco Filho, 1999), neste caso, os resultados representam o vigor de cada lote de sementes e o comportamento fisiológico do genótipo em relação às condições ambientais de realização do teste, sendo que o teste foi realizado sob mesma condição para todas as cultivares. Também Dutra e Teófilo (2007), Mavi e Demir (2007) observaram que lotes de sementes, sendo alguns destes lotes provenientes de genótipos diferentes, com qualidade semelhante diagnosticada através de teste de germinação apresentaram diferenças de qualidade nos testes de vigor. $\mathrm{O}$ teste de germinação não prevê uma avaliação completa da qualidade do lote de sementes (McDonald, 1980).

TABELA 1. Valores médios de massa de 1000 sementes (M 1000 sementes), índice de velocidade de germinação (IVG), teor de água, primeira contagem de germinação ( $1^{\circ}$ Contagem de germinação), germinação e emergência de plantas para sementes de feijão.

\begin{tabular}{|c|c|c|c|c|c|c|}
\hline \multirow[t]{2}{*}{ Cultivares } & $\begin{array}{l}\text { M. } 1000 \\
\text { sementes }\end{array}$ & IVG & $\begin{array}{c}\text { Teor de } \\
\text { água }\end{array}$ & $\begin{array}{l}1^{\text {a }} \text { Contagem de } \\
\text { Germinação }\end{array}$ & Germinação & $\begin{array}{l}\text { Emergência de } \\
\text { plantas }\end{array}$ \\
\hline & $\ldots(g) \ldots$ & & \multicolumn{4}{|c|}{$(\%)$} \\
\hline LP 04-92 & 191,5 & $20,00 \mathrm{~A}$ & 12,25 & $100,00 \mathrm{~A}$ & $100,00 \mathrm{~A}$ & $75,00 \mathrm{~A}$ \\
\hline Juriti & 181,5 & $19,75 \mathrm{~A}$ & 11,65 & $99,74 \mathrm{~A}$ & $99,87 \mathrm{~A}$ & $63,00 \mathrm{C}$ \\
\hline Talismã & 197,0 & $20,00 \mathrm{~A}$ & 11,45 & $100,00 \mathrm{~A}$ & $100,00 \mathrm{~A}$ & $62,00 \mathrm{D}$ \\
\hline Colibri & 202,5 & $20,00 \mathrm{~A}$ & 11,85 & $100,00 \mathrm{~A}$ & $100,00 \mathrm{~A}$ & $62,00 \mathrm{D}$ \\
\hline Pérola & 269,0 & $19,50 \mathrm{~A}$ & 13,85 & $98,99 \mathrm{~A}$ & $99,63 \mathrm{~A}$ & $67,75 \mathrm{~B}$ \\
\hline Rubi & 259,0 & $19,75 \mathrm{~A}$ & 12,00 & $99,87 \mathrm{~A}$ & $99,87 \mathrm{~A}$ & $50,00 \mathrm{~F}$ \\
\hline IAC-Carioca & 224,5 & $19,50 \mathrm{~A}$ & 13,25 & $98,53 \mathrm{~A}$ & $99,50 \mathrm{~A}$ & $60,00 \mathrm{E}$ \\
\hline $\mathrm{CV}(\%)$ & & 2,08 & & 4,74 & 3,62 & 0,23 \\
\hline DMS (1\%) & & 1,19 & & 12,00 & 9,27 & 0,35 \\
\hline
\end{tabular}

* Médias seguidas pela mesma letra, na coluna, não diferem entre si pelo teste de Tukey a 1\% de probabilidade.

$\mathrm{Na}$ tabela 3 são apresentados os teores de água das sementes das amostras após exposição ao teste não sendo observadas diferenças superiores a 4\% (Tabela 2), o que é desejável segundo Marcos Filho (1999), comprovando a uniformidade de condições na execução do teste. Observou-se um aumento do teor de água em função do tempo de exposição. De acordo com Scappa Neto et al. (2001) em estudo a respeito dos equipamentos utilizados para realização do teste de envelhecimento acelerado, sendo o teste realizado a $41{ }^{\circ} \mathrm{C}$, a umidade relativa do ar (UR) dentro das caixas plásticas de envelhecimento é sempre superior a $90 \%$ e os menores valores de UR ocorrem sempre nas primeiras horas de envelhecimento $(0-12 \mathrm{~h})$ independente do teor inicial de água das sementes.

Quanto ao teste de envelhecimento acelerado as amostras diferiram quando expostas às diferentes temperaturas e períodos de exposição, não apresentando diferenças estatísticas apenas quando expostas a $45{ }^{\circ} \mathrm{C}$ pelos períodos de 12 e $24 \mathrm{~h}$ (Tabela 3). Em geral, dentro de cada temperatura testada foi observado declínio na germinação, aumento da taxa de deterioração, com o aumento do período de exposição ao teste, fato também observado por Santos et al. (2004) e Binotti et al. (2008). A duração do processo de deterioração é determinada principalmente, pela interação entre herança genética, o grau de umidade da semente e a temperatura (Delouche, 2002). 
TABELA2. Teor de água de sementes de feijão após exposição a 5 períodos e 3 temperaturas do teste de envelhecimento acelerado.

\begin{tabular}{|c|c|c|c|c|c|}
\hline \multirow{3}{*}{ Cultivares } & \multicolumn{5}{|c|}{$41^{\circ} \mathrm{C}$} \\
\hline & $12 \mathrm{~h}$ & $24 \mathrm{~h}$ & $36 \mathrm{~h}$ & $48 \mathrm{~h}$ & $60 \mathrm{~h}$ \\
\hline & \multicolumn{5}{|c|}{$\%$} \\
\hline LP 04-92 & 19,66 & 24,45 & 24,04 & 26,23 & 30,35 \\
\hline Juriti & 19,33 & 23,31 & 24,10 & 26,83 & 30,18 \\
\hline Talismã & 23,30 & 23,18 & 21,82 & 28,82 & 27,48 \\
\hline Colibri & 19,29 & 20,62 & 23,40 & 26,87 & 26,26 \\
\hline Pérola & 20,68 & 24,96 & 25,01 & 28,63 & 26,78 \\
\hline Rubi & 23,68 & 20,42 & 24,58 & 28,85 & 28,67 \\
\hline \multirow[t]{2}{*}{ IAC-Carioca } & 20,63 & 23,41 & 23,98 & 29,77 & 26,52 \\
\hline & \multicolumn{5}{|c|}{$43^{\circ} \mathrm{C}$} \\
\hline LP 04-92 & 19,20 & 17,02 & 26,96 & 23,20 & 30,48 \\
\hline Juriti & 23,88 & 21,63 & 22,86 & 26,58 & 31,25 \\
\hline Talismã & 22,39 & 17,54 & 22,48 & 27,22 & 32,74 \\
\hline Colibri & 19,34 & 21,61 & 24,86 & 27,24 & 28,94 \\
\hline Pérola & 21,24 & 20,90 & 22,63 & 23,52 & 29,94 \\
\hline Rubi & 21,61 & 21,55 & 26,14 & 23,02 & 29,96 \\
\hline \multirow[t]{2}{*}{ IAC-Carioca } & 20,57 & 18,54 & 23,52 & 23,95 & 30,30 \\
\hline & \multicolumn{5}{|c|}{$45^{\circ} \mathrm{C}$} \\
\hline LP 04-92 & 20,74 & 24,24 & 27,95 & 24,68 & 30,71 \\
\hline Juriti & 20,27 & 24,18 & 26,20 & 28,16 & 29,88 \\
\hline Talismã & 18,83 & 23,71 & 27,74 & 28,55 & 30,26 \\
\hline Colibri & 18,39 & 23,67 & 25,68 & 26,54 & 29,69 \\
\hline Pérola & 18,53 & 20,12 & 23,99 & 24,58 & 26,72 \\
\hline Rubi & 18,37 & 20,01 & 25,50 & 24,69 & 26,84 \\
\hline IAC-Carioca & 20,04 & 23,44 & 25,72 & 26,73 & 27,49 \\
\hline
\end{tabular}


TABELA 3. Resultados do teste de envelhecimento acelerado, para três temperaturas e cinco períodos de exposição ao teste, para sementes de feijão.

\begin{tabular}{|c|c|c|c|c|c|}
\hline \multirow{3}{*}{ Cultivares } & \multicolumn{5}{|c|}{$41^{\circ} \mathrm{C}$} \\
\hline & $12 \mathrm{~h}$ & $24 \mathrm{~h}$ & $36 \mathrm{~h}$ & $48 \mathrm{~h}$ & $60 \mathrm{~h}$ \\
\hline & \multicolumn{5}{|c|}{$\%$} \\
\hline LP 04-92 & $98,87 \mathrm{~A}$ & $99,49 \mathrm{~A}$ & $97,08 \mathrm{AB}$ & $99,50 \mathrm{~A}$ & $94,08 \mathrm{AB}$ \\
\hline Juriti & $89,51 \mathrm{C}$ & $94,61 \mathrm{AB}$ & $91,02 \mathrm{~B}$ & $87,08 \mathrm{CD}$ & $85,25 \mathrm{~B}$ \\
\hline Talismã & $94,61 \mathrm{ABC}$ & $97,48 \mathrm{AB}$ & $95,67 \mathrm{AB}$ & $97,56 \mathrm{AB}$ & $97,56 \mathrm{~A}$ \\
\hline Colibri & $99,26 \mathrm{~A}$ & $98,16 \mathrm{AB}$ & $99,50 \mathrm{~A}$ & $97,80 \mathrm{AB}$ & $97,54 \mathrm{~A}$ \\
\hline Pérola & $94,53 \mathrm{ABC}$ & $91,59 \mathrm{~B}$ & $98,50 \mathrm{AB}$ & $91,52 \mathrm{BC}$ & 89,02 B \\
\hline Rubi & $91,13 \mathrm{BC}$ & $95,05 \mathrm{AB}$ & $94,61 \mathrm{AB}$ & $74,50 \mathrm{D}$ & $83,51 \mathrm{~B}$ \\
\hline IAC-Carioca & $97,56 \mathrm{AB}$ & $96,86 \mathrm{AB}$ & $98,16 \mathrm{AB}$ & $94,61 \mathrm{ABC}$ & $93,03 \mathrm{AB}$ \\
\hline \multirow[t]{2}{*}{$\mathrm{CV} \%$} & 3,89 & 5,56 & 4,63 & 4,59 & 4,69 \\
\hline & \multicolumn{5}{|c|}{$43^{\circ} \mathrm{C}$} \\
\hline LP 04-92 & $95,53 \mathrm{AB}$ & $98,16 \mathrm{~A}$ & $98,75 \mathrm{~A}$ & $97,48 \mathrm{~A}$ & $97,08 \mathrm{~A}$ \\
\hline Juriti & $62,53 \mathrm{C}$ & $90,83 \mathrm{AB}$ & $71,10 \mathrm{BC}$ & 81,12 B & $86,51 \mathrm{BCD}$ \\
\hline Talismã & $82,84 \mathrm{BC}$ & $92,15 \mathrm{AB}$ & $83,44 \mathrm{~B}$ & $90,00 \mathrm{AB}$ & $94,61 \mathrm{ABC}$ \\
\hline Colibri & $98,99 \mathrm{~A}$ & $91,28 \mathrm{AB}$ & $92,25 \mathrm{AB}$ & $93,03 \mathrm{AB}$ & $95,12 \mathrm{AB}$ \\
\hline Pérola & 80,04 BC & $90,09 \mathrm{AB}$ & $72,77 \mathrm{~B}$ & $87,81 \mathrm{AB}$ & $76,54 \mathrm{D}$ \\
\hline Rubi & $79,04 \mathrm{BC}$ & $83,01 \mathrm{~B}$ & $43,32 \mathrm{C}$ & $92,25 \mathrm{AB}$ & $82,06 \mathrm{CD}$ \\
\hline IAC-Carioca & $92,58 \mathrm{AB}$ & $96,00 \mathrm{~A}$ & $81,02 \mathrm{~B}$ & $82,99 \mathrm{~B}$ & $94,21 \mathrm{ABC}$ \\
\hline \multirow[t]{2}{*}{$\mathrm{CV} \%$} & 8,44 & 5,71 & 9,30 & 7,35 & 5,59 \\
\hline & \multicolumn{5}{|c|}{$45^{\circ} \mathrm{C}$} \\
\hline LP 04-92 & $98,16 \mathrm{~A}$ & $89,27 \mathrm{~A}$ & $78,08 \mathrm{BC}$ & $57,53 \mathrm{BC}$ & $57,01 \mathrm{~B}$ \\
\hline Juriti & $94,32 \mathrm{~A}$ & $99,70 \mathrm{~A}$ & $69,82 \mathrm{BCD}$ & $67,13 \mathrm{BC}$ & $26,44 \mathrm{C}$ \\
\hline Talismã & $96,13 \mathrm{~A}$ & $94,53 \mathrm{~A}$ & $58,58 \mathrm{D}$ & $50,50 \mathrm{C}$ & $27,50 \mathrm{C}$ \\
\hline Colibri & $96,00 \mathrm{~A}$ & $97,08 \mathrm{~A}$ & $96,13 \mathrm{~A}$ & $94,54 \mathrm{~A}$ & $86,75 \mathrm{~A}$ \\
\hline Pérola & $93,90 \mathrm{~A}$ & $87,68 \mathrm{~A}$ & $81,55 \mathrm{~B}$ & $72,38 \mathrm{~B}$ & $22,76 \mathrm{C}$ \\
\hline Rubi & $91,13 \mathrm{~A}$ & $91,87 \mathrm{~A}$ & 76,71 BCD & $52,00 \mathrm{BC}$ & $27,25 \mathrm{C}$ \\
\hline IAC-Carioca & $96,68 \mathrm{~A}$ & $94,88 \mathrm{~A}$ & $59,54 \mathrm{CD}$ & $56,57 \mathrm{BC}$ & $27,17 \mathrm{C}$ \\
\hline $\mathrm{CV} \%$ & 6,08 & 6,15 & 6,89 & 7,77 & 10,49 \\
\hline
\end{tabular}

* Médias seguidas pela mesma letra, na coluna, não diferem entre si pelo teste de Tukey a 1\% de probabilidade.

Quando da aplicação do teste à temperatura de $45{ }^{\circ} \mathrm{C}$ observou-se menores valores de germinação principalmente a partir de $36 \mathrm{~h}$ de exposição ao teste, fato observado também por Paiva (2008) em sementes de forrageira.

Com relação ao comportamento dos genótipos é possível detectar que há materiais mais sensíveis aos efeitos da temperatura e tempo de exposição ao teste e também materiais mais resistentes. Isto sugere que para alguns materiais há necessidade de se estudar outras temperaturas e períodos de exposição das sementes de forma a avaliar o seu vigor com segurança e adequadamente, ou seja, pode ser mais adequado agrupar alguns cultivares para indicar a melhor temperatura e período de exposição.

A $45^{\circ} \mathrm{C}$ observamos uma brusca queda de porcentagem de germinação para as cultivares Juriti, Talismã e IAC Carioca após 36 horas de exposição, para Rubi após 48 horas e para Pérola após 60 horas, sendo que a cultivar Colibri apresenta-se como mais resistente às condições de alta temperatura em relação aos demais genótipos. Com 
relação à coloração do tegumento não se pode afirmar relação deste fator com os parâmetros envolvidos no teste, pois a linhagem LP 04-92 e a cultivar Talismã que possuem tegumento preto apresentaram comportamento semelhante aos outros genótipos.

Por outro lado, do ponto de vista prático, essa maior resistência de algumas cultivares às condições de alta temperatura e alta umidade é um fator favorável para indicar a semeadura destas cultivares em locais onde ocorrem estas condições garantindo uma emergência adequada e evitando perdas e aumento nos custos de produção em função dos gastos com nova semeadura.

Embora os genótipos apresentem diferenças no comportamento, a comparação entre os mesmos tem a única finalidade de identificar aqueles que apresentam uma maior ou menor sensibilidade a uma determinada situação. Assim, de posse dessas informações pode-se fazer recomendações mais seguras no que se diz respeito a condições mais adequadas para a avaliação do vigor e também para semeadura, uma vez que estes materiais são cultivados em vários Estados brasileiros, os quais, de modo geral apresentam diferentes condições climáticas.

$\mathrm{Na}$ Tabela 4 são apresentados os resultados de correlação linear entre envelhecimento acelerado, germinação e vigor. Observa-se correlação linear positiva entre a emergência de plantas e o teste de envelhecimento acelerado realizado a $41^{\circ} \mathrm{C} / 12 \mathrm{~h}$ e $48 \mathrm{~h}, 43^{\circ} \mathrm{C} / 24 \mathrm{~h}$ e $36 \mathrm{~h} \mathrm{e}$ $45^{\circ} \mathrm{C} / 12 \mathrm{~h}$. Dentre estes a realização do teste a $41^{\circ} \mathrm{C} / 48 \mathrm{~h}$ e $43{ }^{\circ} \mathrm{C} / 24 \mathrm{~h}$ e $36 \mathrm{~h}$ foi significativo a $1 \%$ de probabilidade com os maiores valores de $r, r=0,70, r=0,62$ e $r=0,71$, respectivamente, e correspondem com maior fidelidade aos resultados observados para emergência de plantas.

TABELA 4. Resultados de correlação linear entre o teste de envelhecimento acelerado, germinação e vigor.

\begin{tabular}{|c|c|c|c|c|c|}
\hline Temperaturas & Períodos & $\begin{array}{c}1^{\circ} \text { Contagem de } \\
\text { Germinação }\end{array}$ & $\begin{array}{c}\text { Velocidade de } \\
\text { Germinação }\end{array}$ & Germinação & $\begin{array}{c}\text { Emergência de } \\
\text { Plantas }\end{array}$ \\
\hline \multirow{5}{*}{$41^{\circ} \mathrm{C}$} & $12 \mathrm{~h}$ & 0,1360 & 0,1441 & 0,1518 & $0,4415^{*}$ \\
\hline & $24 \mathrm{~h}$ & 0,2542 & 0,3123 & 0,3079 & 0,2875 \\
\hline & $36 \mathrm{~h}$ & $-0,0598$ & 0,0767 & 0,0906 & 0,1844 \\
\hline & $48 \mathrm{~h}$ & 0,2229 & 0,2427 & 0,2479 & $0,7011 * *$ \\
\hline & $60 \mathrm{~h}$ & 0,2975 & 0,3280 & 0,3256 & 0,3075 \\
\hline \multirow{5}{*}{$43^{\circ} \mathrm{C}$} & $12 \mathrm{~h}$ & 0,2144 & 0,2599 & 0,2622 & 0,2490 \\
\hline & $24 \mathrm{~h}$ & $-0,0365$ & 0,0299 & 0,0300 & $0,6288 * *$ \\
\hline & $36 \mathrm{~h}$ & 0,1979 & 0,2207 & 0,1941 & $0,7116^{* *}$ \\
\hline & $48 \mathrm{~h}$ & 0,3339 & 0,1916 & 0,2248 & 0,2261 \\
\hline & $60 \mathrm{~h}$ & 0,2656 & 0,3009 & 0,2425 & 0,3131 \\
\hline \multirow{5}{*}{$45^{\circ} \mathrm{C}$} & $12 \mathrm{~h}$ & $-0,2306$ & $-0,1551$ & $-0,1391$ & $0,4376 * *$ \\
\hline & $24 \mathrm{~h}$ & 0,0282 & 0,0210 & 0,0442 & $-0,2509$ \\
\hline & $36 \mathrm{~h}$ & 0,2832 & 0,2289 & 0,2507 & 0,1069 \\
\hline & $48 \mathrm{~h}$ & 0,1010 & 0,1055 & 0,1365 & 0,1290 \\
\hline & $60 \mathrm{~h}$ & $0,4067 *$ & $0,4002 *$ & $0,3970 *$ & 0,2374 \\
\hline
\end{tabular}

**, *, significativo pelo teste $t$ a 1 e $5 \%$ de probabilidade respectivamente.

A realização do teste à temperatura de $43{ }^{\circ} \mathrm{C}$ permite a obtenção do resultado por um menor período de tempo, com $24 \mathrm{~h}$ de antecedência em relação a realização do teste a $41^{\circ} \mathrm{C} / 48 \mathrm{~h}$.

O envelhecimento acelerado está correlacionado com as taxas de germinação e emergência de raiz, bem como com a taxa de emergência no campo (Kulik e Yalik,
1982). Também Contreras e Barros (2005) e Anfinrud e Schneider (1984) observaram correlação linear positiva entre o teste de envelhecimento acelerado e a emergência de plantas em campo para sementes de alface e girassol respectivamente.

Houve correlação linear positiva a $5 \%$ entre o teste de envelhecimento acelerado a $45^{\circ} \mathrm{C} / 60 \mathrm{~h}$ e primeira contagem 
de germinação $(\mathrm{r}=0,40)$, velocidade de germinação $(\mathrm{r}=0,40)$ e germinação $(\mathrm{r}=0,39)$, no entanto a correlação não foi significativa em relação à emergência de plantas. Um teste de vigor deve entre outros correlacionar-se com o desempenho em campo (McDonald, 1980) e por este motivo a correlação significativa entre o envelhecimento acelerado a $45{ }^{\circ} \mathrm{C} / 60 \mathrm{~h}$ e germinação, primeira contagem de germinação e velocidade de germinação não torna adequada a realização do teste sob estes parâmetros.

\section{CONCLUSÕES}

Sementes de feijão com qualidade semelhante no teste de germinação apresentam níveis distintos de qualidade pelo teste de envelhecimento acelerado.

O teste de envelhecimento acelerado para sementes de feijão pode ser realizado com aplicação de maior temperatura e redução do período de exposição ao teste, ou seja, $43{ }^{\circ} \mathrm{C} / 24 \mathrm{~h}$.

\section{REFERÊNCIAS}

ANFINRUD, M.N.; SCHENEITER, A.A. Relationship of sunflower germination and vigor tests to field performance. Crop Science, v.24, p.341-344, 1984.

BANZATTO, D.A.; KRONKA, N. Experimentação agrícola. 4.ed. Jaboticabal: Funep, 2006. 237p.

BINOTTI, F.F.S.; HAGA, K.I.; CARDOSO, E.D.; ALVES, C.Z.; SÁ, M.E.; ARF, O. Efeito do período de envelhecimento acelerado no teste de condutividade elétrica e na qualidade fisiológica de sementes de feijão. Acta Scientiarum, v.30, n.2, p.247-254, 2008.

BRASIL. Ministério da Agricultura, Pecuária e Abastecimento. Regras para análise de sementes. Ministério da Agricultura, Pecuária e Abastecimento. Secretaria de Defesa Agropecuária. Brasília, DF: Mapa/ ACS, 2009. 395p.

CONAB. Acompanhamento da safra brasileira. Disponível em: <http://www.conab.gov.br/conabweb/ download/safra/1graos_09.10.pdf $>$. Acesso em: 1 mar. 2010 .

CONTRERAS, S.; BARROS, M. Pruebas de vigor en semillas de Lechuga y su correlación con emergencia. Ciência y Investigacion Agrária, v.32, n.1, p.3-11, 2005.

COPELAND, L.O.; McDONALD, M.B. Principles of seed science and technology, Kluwer Massachusets: Academic
Publishers, 4.ed. p.165-192, 2001.

DELOUCHE, J. Germinação, deterioração e vigor da semente. Seed News, n.6, p.24-31, 2002.

DIAS, D.C.S.F., MARCOS FILHO, J. Testes de vigor baseados na permeabilidade das membranas celulares: II. Lixiviação de potássio. Informativo ABRATES, v.5, n.1, p.37-41, 1995.

DUTRA, A.S.; TEÓFILO, E.M. Envelhecimento acelerado para avaliar o vigor de sementes de feijão caupi. Revista Brasileira de sementes, v.29, n.1, p.193-197, 2007.

HALMER, P. Commercial seed treatment technology. In: BLACK, M. and BEWLEY, J.D. (Ed.) Seed Techonology and its Biological Basics. England: Sheffield Academic Press, 2000, p.266-273.

KULIK, M.M.; YALICH, R.W. Evolution of vigor tests in soybean seeds: relationship ofaccelerated aging, cold, sand bench and speed of germination tests to Field performance. Crop Science, v.22, p.766-700, 1982.

MAGUIRE, J.D. Apeed of germination-aid in selection and evalution for seedling emergence and vigor. Crop Science, v.2, n.2, p.176-177, 1962.

MARCOS FILHO, J. Teste de Envelhecimento Acelerado. In.: KRYZANOWSKI, F.C.; VIEIRA, R.D.; FRANÇA NETO, J. DE B. (Ed.) Vigor de sementes: conceitos e testes. Londrina: ABRATES, 1999, p.3.1-3.24.

MARCOS FILHO, J. Teste de envelhecimento acelerado. In: VIEIRA, R.D.; CARVALHO, N.M. (Ed.). Testes de vigor em sementes. Jaboticabal: Funep, 1994. p.133-149.

MAVI, K.; DEMIR, I. Controlled deterioration and accelerated aging tests predict relative seedling emergence potential of melpon seed lots. HortScience, v.42, p.14311435, 2007.

McDONALD, M.B. Assessment of seed quality. Horticultural Science, v.15, p.784-788, 1980.

MELLO, V.D.C.; TILLMANN, M.A. O teste de vigor em câmara de envelhecimento precoce. Revista Brasileira de Sementes, v.9, n.2, p.93-102, 1987.

PAIVA, A.S.; RODRIGUES, T.J.D.; CANCIAN, A.J.; LOPES, M.M.; FERNANDES, A.C. Qualidade física e fisiológica de sementes da leguminosa forrageira Macrotyloma acillare cv. Java. Revista Brasileira de Sementes, v.30, n.2, p.130-136, 2008.

RENGEL, E.P. Produção de sementes de feijoeiro comum no Brasil. In: VIII CONGRESSO NACIONAL DE PESQUISA DE FEIJÃO, 8., 2005, Goiânia, Anais... Santo 
Antônio de Goiás: Embrapa Arroz e Feijão, 2005. p.12571261.

SÁ, M.E. Importância da adubação na qualidade de sementes. In: SÁ, M.E.; BUZZETI, S. (Coord.), Importância da adubação na qualidade dos produtos agrícolas. São Paulo: Ícone, 1994, 437p.

SANTOS, C.M.R.; MENEZES, N.L.; VILLELA, F.A. Alterações fisiológicas e bioquímicas em sementes de feijão envelhecidas artificialmente. Revista Brasileira de Sementes, v.26, n.1, p.110-119, 2004.
SCAPPA NETO, A.; BITTENCOURT, S.R.M.; VIEIRA, R.D.; VOLPE, C.A. Efeito do teor inicial de água de sementes de feijão e da câmara no teste de envelhecimento acelerado. Scientia Agrícola, v.58, p.747-751, 2001.

YOKOYAMA, L.P.; WETZEL, C.T.; VIEIRA, E.H.N.; PEREIRA, G.V. Sementes de feijão: Produção, uso e comercialização. In: VIEIRA, E.H.N.; RAVA, C.A. (Ed.). Sementes de Feijão: produção e tecnologia. Goiás: EMBRAPA Arroz e feijão, 2000. p.249-270.

ZONTA, E.P.; MACHADO, A.A. SANEST: sistema de análise de variância por microcomputadores. Pelotas: UFPel, 1991. 